\title{
Systemic Inflammation in COPD is not influenced by pulmonary rehabilitation
}

\section{$\underline{\text { ABSTRACT }}$}

Background: Pulmonary Rehabilitation (PR) is known to lead to improvements in exercise tolerance, health related quality of life and help reduce symptoms. Exercise, although of great benefit, can increase the inflammatory response related to Chronic Obstructive Pulmonary Disease, depending on intensity and duration. Through this study, the effects of a 12 week, high intensity PR programme on COPD inflammatory related markers were investigated. Method: Sixty COPD patients were enrolled, 49 of which completed the programme. A two hour high intensity PR programme was delivered, twice weekly for 12 weeks. The following markers were assessed at baseline, 4, 8 and 12 weeks through rehabilitation - C Reactive Protein (CRP), Erythrocyte Sedimentation Rate (ESR), Neutrophil, Eosinophil counts, complete blood count (CBC), six minute walk test (6MWT) and St. George's Respiratory Questionnaire (SGRQ). Serum Amyloid A levels were assessed at baseline, week 8 and 12 and exhaled $\mathrm{NO}$ at baseline and upon completion of the programme.

Results: This 12 week PR programme resulted in significant improvements in both the $6 \mathrm{MW}$ distance and health quality of life, but resulted in no changes in the inflammatory markers.

Conclusion: Beneficial effects on functional and HR-QoL measures resulted, which however appear unrelated to changes in the systemic inflammatory markers.

Key Words: $\quad$ Exercise, Intensity, Duration, Systemic Inflammation 